\title{
Multilinguales
}

\section{Pour une didactisation des nouvelles de Femmes d'Alger dans leur appartement d'Assia Djebar}

For a Didactization of the Short Stories "Algiers Women in their Apartment"

by Assia Djebar

\section{M'hand AMMOUDEN}

\section{CpenEdition}

\section{Journals}

Édition électronique

URL : http://journals.openedition.org/multilinguales/878

DOI : $10.4000 /$ multilinguales. 878

ISSN : 2335-1853

Éditeur

Université Abderrahmane Mira - Bejaia

Référence électronique

M'hand AMMOUDEN, « Pour une didactisation des nouvelles de Femmes d'Alger dans leur appartement d'Assia Djebar », Multilinguales [En ligne], 6 | 2015, mis en ligne le 01 décembre 2015, consulté le 17 septembre 2019. URL : http://journals.openedition.org/multilinguales/878 ; DOI : 10.4000/ multilinguales. 878

Ce document a été généré automatiquement le 17 septembre 2019

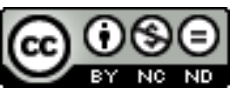

Multilinguales est mise à disposition selon les termes de la Licence Creative Commons Attribution Pas d'Utilisation Commerciale - Pas de Modification 4.0 International 


\title{
Pour une didactisation des nouvelles de Femmes d'Alger dans leur appartement d'Assia Djebar
}

\author{
For a Didactization of the Short Stories "Algiers Women in their Apartment" \\ by Assia Djebar
}

M'hand AMMOUDEN

1 Notre étude s'inscrit dans la didactique des littératures francophones dont l'un des principaux objectifs et intérêts est de faire des propositions susceptibles de conférer une meilleure place à ces littératures à côté de la littérature française dans les pratiques de classe.

Il s'agit globalement de s'interroger sur la possibilité et les avantages du recours aux textes francophones d'auteurs algériens comme supports d'enseignement du français langue étrangère; aussi bien dans le contexte algérien que dans d'autres contextes. Notons à ce sujet qu'on réclame, par exemple, en France, depuis plusieurs années l'enseignement des littératures «étrangères ». En témoigne un appel, Pour l'enseignement des Littératures Francophones en France, ainsi entamé :

Nous, professeurs de lettres de l'enseignement secondaire et de l'enseignement supérieur, écrivains des francophonies littéraires, élus des instances représentatives des établissements d'enseignements (Conseil supérieur de l'Éducation, Conseil National de l'Enseignement Supérieur et de la Recherche...), présidents d'universités, étudiants de lettres, lycéens, amoureux des lettres, nous prononçons pour une extension de l'enseignement des Littératures Francophones en France dans les divers secteurs du système éducatif français au primaire, au secondaire, au supérieur. (...)Une page est désormais tournée, celle de la relégation des littératures francophones dans les périphéries de la littérature, au nom d'une conception restrictive de la littérature française. (Café-Pédagogique, 2006)ํ․

Notre hypothèse est que la didactisation de ces textes est non seulement possible, mais indispensable pour atteindre quelques-unes des recommandations actuelles du Ministère de l'Éducation Nationale (désormais MEN) et de la didactique. Néanmoins, 
cela implique d'effectuer certains choix théoriques parmi ceux, nombreux, qui coexistent aujourd'hui.

Cet article est composé de trois volets :

- le premier, "pour la didactisation des textes d'auteurs algériens », est consacré à l'examen de la possibilité, légitimée par la didactique «institutionnelle » et par celle des chercheurs², d'une utilisation de textes d'auteurs algériens, dans l'enseignement du français et de sa littérature ;

- le deuxième, "pour une didactisation des nouvelles, dans le cadre d'une séquence et/ou d'un projet ", est réservé à une illustration des possibilités de cette didactisation à travers Femmes d'Alger dans leur appartement d'Assia Djebar ; et,

- le troisième, "pour le développement de la compétence plurilingue et pluriculturelle", développe les possibilités et raisons d'attribuer une meilleure place à ce type de texte dans l'enseignement du FLE aussi bien dans le secteur de l'Education Nationale que dans celui de l'enseignement supérieur.

\section{Pour la didactisation des textes d'auteurs algériens}

\subsection{Les embûches d'une didactisation du texte littéraire francophone}

Vouloir faire une étude sur la possibilité de didactiser les œuvres de Djebar, nous place d'emblée dans une problématique plus vaste : celle relative à la didactisation de textes d'auteurs des littératures francophones. Pour traiter de cette problématique, nous commencerons par décrire les contextes institutionnel et théorique dans lesquels nous la situons.

3 En effet, cette étude doit être placée dans le sillage d'une réforme progressive de l'Éducation Nationale, amorcée en 2002. Telle que préconisée, entre autres, dans le Référentiel général des programmes (MEN, 2009 a) et dans le Guide méthodologique d'élaboration des programmes (MEN, 2009 b), cette réforme se traduit, sur le plan didactique, essentiellement par l'approche par les compétences ${ }^{3}$.

On prône aussi les approches curriculaire ${ }^{4}$ et intégrée ${ }^{5}$ et la démarche du projet $^{6}$.

Dans les documents qui concernent l'enseignement des langues, on indique qu'il faut travailler par séquences didactiques ${ }^{7}$.

On recommande par ailleurs aux concepteurs des programmes d'introduire l'enseignement d'auteurs algériens.

Ainsi, en traitant des finalités assignées aux matières du domaine des connaissances en langues et littératures, les auteurs du Guide méthodologique d'élaboration des programmes retiennent l'objectif suivant: "Dans le domaine de la littérature, l'élève devra connaître les auteurs algériens et maghrébins, sans rester fermé aux autres littératures du monde africain et du monde arabe » (MEN, 2009b : 39).

Cette consigne institutionnelle algérienne oblige les concepteurs des programmes et des manuels de français à ne pas se contenter des auteurs français en faisant une place notamment aux auteurs algériens.

Sur le plan théorique, nous retenons en particulier l'intérêt porté, par les théoriciens de l'enseignement des langues aux approches qui préconisent le développement de la compétence plurilingue et pluriculturelle ${ }^{8}$, 
Telle que formulée, non seulement cette recommandation n'est pas réservée à l'approche actionnelle, ni au seul contexte européen, mais elle permet de faire la part belle à la littérature dans la maîtrise de cette compétence.

Mais, au niveau de sa conception didactique, la tâche n'est pas aisée et «la fortune du mot didactique ne doit pas nous abuser. Les recherches sont loin d'avoir investi l'ensemble des champs disciplinaires et le domaine littéraire moins que les autres" (J. M. Rosier) ${ }^{9}$; nous pouvons ajouter que la didactique des littératures francophones l'est aujourd'hui encore moins et est quasi-inexistante.

A cette indigence s'ajoute un autre écueil d'ordre méthodologique: celui de la (re)production par les apprenants du genre enseigné, dans le cadre d'une séquence didactique ${ }^{10}$.

Telle que théorisée en 2002 par De Pietro et Dolz, Noverraz et Schneuwly, la séquence nécessite en effet une production sur le modèle du genre sur lequel elle s'articule au début et à la fin (production initiale - production finale).

Selon De Pietro ${ }^{11}$, après la première phase dite de "mise en situation ", visant surtout à " faire émerger un projet de communication", suit la seconde étape, la "production initiale durant laquelle les élèves sont invités à réaliser une première fois l'activité communicative projetée, dans toute sa complexité» (De Pietro, 2002 : 17-18).

Cette première production, qui a valeur d'évaluation-diagnostic, permet de découvrir les représentations des apprenants et leurs besoins qui seront pris en charge dans une troisième étape à travers des « ateliers » (appelés aussi « modules»). La quatrième étape est celle de

la production finale [qui] se situe ainsi, comme en miroir à la production initiale, à

l'articulation entre les projets de communication et d'apprentissage :

aboutissement $\mathrm{du}$ projet communicatif, elle permet de mettre en œuvre les apprentissages effectués et de les rendre visibles. (idem.)

Ce schéma de la séquence nous interpelle en tant qu'enseignants du français langue étrangère (toujours apprenants), et dans le domaine qui nous intéresse ici, à savoir la didactique du texte littéraire. Est-il concevable que des apprenants, surtout débutants, puissent élaborer un conte, une nouvelle ou un roman, etc., sur le modèle de la notice d'emploi d'un appareil quelconque?

Or, c'est ce vers quoi conduit habituellement l'application du schéma de la séquence didactique $^{12}$, en FLE, en milieu scolaire.

Certes, les concepteurs des nouveaux programmes et manuels, certains inspecteurs de l'Education Nationale recommandent parfois de se contenter, dans ce cas, d'activités de réécriture ou de production partielles (rédiger le début d'une histoire, imaginer une autre fin, etc.).

Mais le problème, sur le terrain, reste posé.

Les concepteurs de l'un des anciens manuels de français de la $3^{\text {ème }}$ année secondaire (Bouchouikha et al. 1989), dont les contenus sont répartis par « dossiers ", suggéraient, quant à eux, une focalisation sur la réception (ou compréhension), dans les deux derniers dossiers intitulés : "Lire la poésie contemporaine» (p.195 264) et "Lire le roman » (pp. 265281$).$

Peut-être, pourrait-on envisager de reconduire cette recommandation en l'adaptant aux séquences didactiques portant sur les genres littéraires qui seraient focalisées sur le développement de compétences de réception; elles se réaliseraient, par conséquent, sans les phases de productions "initiale» et "finale", auxquelles pourrait se substituer le travail exclusif des compétences de compréhension. 
Par ailleurs, si l'examen de certains manuels destinés à l'enseignement du français dans l'Éducation Nationale montre que les textes issus de la littérature algérienne n'y sont pas totalement absents, ils n'y sont pas pour autant suffisamment présents ${ }^{13}$.

De plus, ces textes ne sont pas exploités en tant que tels dans la mesure où ils sont généralement employés uniquement comme des alibis à l'enseignement de faits de langue.

Dans la séquence consacrée à la nouvelle dans le manuel de français destiné aux classes de $3^{\text {ème }}$ AS (Bouchouika et al., 2004: 105 151), par exemple, les extraits des textes d'auteurs algériens sont surtout utilisés pour l'enseignement des pronoms personnels, des adjectifs possessifs, des indicateurs temporels, etc.

Si des textes d'auteurs francophones sont présents dans cette séquence, ce n'est pas toujours le cas dans d'autres, et il ne s'agit pas toujours de textes d'auteurs algériens. Ainsi, dans l'actuel manuel de français de $3^{\text {ème } A S ~(M a h b o u b i, ~ R e k k a b ~ \& ~ A l l a o u i, ~} 2007$ : 165216 ), aucun texte d'un auteur algérien ne figure dans les trois séquences didactiques qui constituent le projet intitulé « Rédiger une nouvelle pour exprimer son imaginaire et provoquer trouble et questionnement chez le lecteur ».

\subsection{La littérature francophone et la notion de « document authentique "}

4 Envisager la didactisation des littératures francophones, sur un plan théorique, conduit à s'interroger sur le statut, requis par les didacticiens, de "documents authentiques » des textes, étant donné qu'ils sont l'œuvre d'auteurs non-natifs de la langue cible. Ajoutons qu'on est confronté aujourd'hui à la coexistence de nombreuses définitions, souvent très nuancées, de la notion de « document authentique ».

Au milieu des années soixante-dix, dans Dictionnaire de didactique des langues (1976: 59), le « document authentique » était défini comme «tout document, sonore ou écrit, qui n'a pas été conçu expressément pour la classe ou pour l'étude de la langue, mais pour répondre à une fonction de communication, d'information, ou d'expression linguistique réelle ».

Robert Bouchard ${ }^{14}$ formule à ce sujet une recommandation :

L'adjectif « authentique » ne doit (...) pas être pris au pied de la lettre. Son intérêt est seulement de permettre une opposition simple entre deux types de ressources langagières pour la classe de langue, les documents authentiques donc et les documents pédagogiques, fabriqués de manière purement ad hoc.

En outre, le recours au "document authentique », de façon générale, a une finalité, celle entre autres, de " conduire à une communication plus vraisemblable en langue-cible et $\grave{a}$ une familiarisation plus directe avec la culture-cible» (Defays et Deltour, 2003: 263); et le recours à la littérature en tant que document authentique, en particulier «apporte beaucoup à la motivation des apprenants et à leur compréhension de la culture- cible " (idem : 109).

Dans le cas du français, un tel document en littérature impliquerait, d'emblée, de se limiter au choix exclusif de textes écrits par des Français pour une (meilleure) compréhension de la civilisation française (« culture-cible»).

Cependant, certains didacticiens dont Adami $\mathrm{H}$. soulignent que "l'accès direct au document brut peut effectivement parfois poser problème » (Adami, 2009: 170) au niveau de leur compréhension par les apprenants débutants (non natifs). 
De plus, si cette définition du document authentique comme produit exclusivement par des natifs peut s'avérer appropriée dans une approche communicative, la première à avoir encouragé le recours à ces documents, elle n'est guère conforme aux principes des approches plus récentes telles que l'approche par les compétences et l'approche actionnelle.

A ce sujet, Puren (2009) explique que si l'objectif social de référence, avec l'approche communicative, était de " préparer les élèves à rencontrer occasionnellement des natifs de la langue-culture étrangère (au cours de voyages ponctuels, en particulier de tourisme)» (p. 30), celui qui sera retenu à partir des années 90 est «celui de préparer les élèves à cohabiter harmonieusement dans une société qui est de plus en plus multilingue et multiculturelle» (p. 31) ${ }^{15}$. Il ajoute qu'avec la perspective actionnelle, l'objectif social de référence mis en valeur est celui qui consiste à

préparer les apprenants à travailler, dans leur propre pays ou dans un pays étranger, avec des natifs de différentes langues-cultures étrangères, comme c'est déjà le cas par exemple dans des entreprises multinationales, ou dans des Universités où des étudiants de tous pays étudient ensemble dans la langue du pays d'accueil. (p. 31)

Rappelons que les approches actionnelle ${ }^{16}$ et par compétences impliquent qu'on tienne compte des caractéristiques de l'environnement dans lequel vit l'apprenant. Intervenant dans le cadre de la réforme de l'enseignement en Algérie, Mohammed Miled (2005: 127) ${ }^{17}$ décrit le contexte qui détermine les «facteurs d'une réécriture et d'une refonte d'un curriculum » et précise que l'

On assiste actuellement à un changement de paradigme éducatif qui insiste davantage sur le processus d'apprentissage. Cela signifie que l'élaborateur du programme et l'enseignant accordent un intérêt particulier à l'élève, autrement dit à ses besoins et à son environnement, à son imaginaire, à son rythme d'apprentissage ainsi qu'à ses stimulations et ses motivations. (Idem.)

Cet «intérêt particulier accordé à l'élève », dans ce contexte, implique donc de privilégier les documents issus de son environnement immédiat. C'est d'ailleurs l'une des sept recommandations retenues par Ahcène Lagha, en tant que directeur général de l'office national des publications scolaires (ONPS), pour l'élaboration des contenus des nouveaux manuels de cette "refonte de la pédagogie en Algérie »: "Le manuel doit véhiculer un contenu socioculturel en rapport avec l'environnement de l'apprenant en mesure de promouvoir la culture de la lecture » (Idem. : 59).

Ajoutons que la source même du "document authentique", à savoir le «locuteur natif » que présupposent certaines conceptions, est une notion devenue suspecte dans l'optique de "la compétence plurilingue et de la didactique des langues étrangères », comme le souligne Daniel Coste $(2010: 150)$ :

Définie comme à la fois une et plurielle, elle [la compétence plurilingue] se présente comme capacité à gérer et à étendre un répertoire de langues et variétés maîtrisées à des degrés divers. Il s'agit de sortir de représentations et de pratiques reposant sur les images mythiques du natif (Renaud, 1998) ${ }^{18}$ et $d u$ "parfait bilingue" et sur des modèles de l'apprentissage de plusieurs langues comme d'un cumul de compétences à la fois homologues et bien séparées.

Cette conception du " document authentique ", à l'instar de celle de "locuteur natif » appelé aussi « locuteur national » (M. Byram et al., 2002) - est en porte-à-faux avec les approches qui visent le développement de la compétence plurilingue et pluriculturelle. C'est ainsi que celle-ci s'en est trouvée assouplie dans sa formulation, comme dans le Dictionnaire de didactique du français langue étrangère et seconde (Cuq, dir., 2003 : 29) :

La caractérisation d' "authentique ", en didactique des langues, est généralement associée à « document » et s'applique à tout message élaboré par des francophones 
pour des francophones à des fins de communication réelle : elle désigne donc tout ce qui n'est pas conçu à l'origine pour la classe.

Nous pouvons donc considérer que les textes des littératures francophones, même s'ils ne sont pas élaborés par des natifs pour des natifs, sont des documents authentiques dans le sens retenu à la fois par Robert Bouchard (cf. supra) et dans celui du Dictionnaire de didactique du français langue étrangère et seconde (cf. supra).

Ce sont donc des documents élaborés par des francophones pour des francophones, à des fins de communication (littéraire) réelle, puisque non «fabriqués " pour classe.

Ils « apportent beaucoup à la motivation des apprenants et à leur compréhension de la culturecible» (Defays et Deltour, 2003 : 109) et de la "culture-source », telles que convoquées en texte; d'où leur intérêt dans le développement de la compétence plurilingue et pluriculturelle.

\subsection{Littérature algérienne : support didactique productif}

Sur le plan de la didactique institutionnelle, notre objet d'étude nous amène à nous intéresser aux finalités assignées à l'École algérienne, et à la place accordée aux littératures francophones.

L'institution ministérielle algérienne fixe l'objectif de l'enseignement des langues en ces termes: l'élève doit «connaître, par l'intermédiaire des textes, les noms des écrivains algériens, maghrébins et arabes, et d'autres écrivains du monde les plus connus appartenant à la littérature universelle » (MEN, 2009b : 46).

Ce recours exigé aux textes littéraires algériens et autres est d'ailleurs indispensable pour la réalisation d'un autre objectif assigné à l'enseignement des langues étrangères : «[Les langues étrangères] sont enseignées en tant qu'outil de communication permettant l'accès direct à la pensée universelle en suscitant des interactions fécondes avec les langues et cultures nationales » (ibid. : p. 26).

Or, ces interactions ne seraient pas envisageables sans la littérature nationale en tant que partie intégrante de la culture. Cela remettrait en question la possibilité de s'appuyer sur les acquis de chacune des langues et cultures concernées pour favoriser l'apprentissage de l'autre et celle d'une confrontation des caractéristiques des deux.

Il est pourtant très possible que de telles démarches, favorisent les approches curriculaire et intégrée, prônées par le Ministère de l'éducation (cf. supra). Cette possibilité résulterait de la nature des thèmes, des aspects textuels (mélange de langues, traduction, etc.) et culturels véhiculés par cette littérature, comme nous l'illustrerons dans la troisième partie de cet article.

Soulignons à ce sujet que les littératures francophones, justement, "utilisent le français comme langue, mais pour exprimer leur propre culture et civilisation, différente de l'hexagonale " (Brahimi, 2001). Leur utilisation permettra donc de pratiquer les approches contrastives, de faire découvrir les similitudes et les différences et d'établir des passerelles entre les langues-cultures locales et étrangères. De plus, les approches centrées sur l'apprenant (dont l'approche par les compétences), impliquent le recours à des textes issus de l'environnement immédiat de celui-ci, surtout quand on s'inscrit dans la logique des curricula, comme c'est le cas en Algérie (cf. supra).

6 Celles-ci impliquent, entre autres, la prise en compte des spécificités du contexte de l'apprenant, dont celles relatives aux pratiques langagières et culturelles. En effet, une analyse des besoins des apprenants, surtout dans les contextes de langues étrangères et 
secondes, implique l'inventaire et l'étude des caractéristiques des genres textuels auxquels ils ont affaire d'abord dans leur environnement immédiat. Les caractéristiques de ces genres peuvent d'ailleurs être nuancées d'une société à une autre :

Les genres du discours sont des produits culturels, propres à une société donnée, élaborés au cours de son histoire et relativement stabilisés. Ils régissent les discours des membres de cette société engagés dans les infinies situations de la vie sociale (Reuter et al. $2010: 117$ ).

Que l'objectif soit d'ailleurs l'enseignement de la langue ou celui de la littérature, ces théories nous conduiraient, surtout avec des débutants, à privilégier au moins dans un premier temps des documents traitant de leur contexte, de leur histoire et de leurs cultures parce qu'ils sont censés s'inscrire dans leur Zone de Développement Proche $(\mathrm{ZDP})^{19}$, contrairement à ceux produits par des étrangers et qui traitent d'autres contextes.

Le recours à cette littérature est d'ailleurs un des moyens d'assurer le transfert des connaissances, selon le processus prôné par Tardif et Meirieu (1996) et encore reconnu aujourd'hui (Rosier, 1997), de « contextualisation, décontextualisation, recontextualisation »:

Le transfert se produit lorsqu'une connaissance acquise dans un contexte particulier peut être reprise d'une façon judicieuse et fonctionnelle dans un nouveau contexte, lorsqu'elle peut être recontextualisée. Cependant, il importe de signaler que, pour pouvoir recontextualiser une connaissance, l'élève doit y avoir accès en mémoire. C'est là une condition incontournable pour que le transfert soit possible. ${ }^{20}$

C'est cet "accès en mémoire " que favoriseraient, en principe, les textes d'auteurs algériens pour les apprenants en Algérie parce que, comme l'expliquera plus tard Rosier (1997 : 100),

Le passage à l'abstraction commence par la recherche d'exemples empruntés à un environnement précis affectif et cognitif, celui de l'apprenant. (...) Enfin la didactique du français a mis en évidence l'importance de l'acquisition de connaissances en milieu naturel.

Quelques lignes plus loin, l'auteur précise, dans le cadre de l'enseignement littéraire en Belgique :

Or, l'enseignement de la littérature fait peu ou jamais référence au contexte régional d'origine des élèves. Cette déréalisation est d'autant plus forte à la périphérie francophone, puisqu'on y enseigne la culture patrimoniale française en convoquant une structuration historique et chronologique qui n'a de sens que pour l'identité nationale française. ${ }^{21}$

C'est précisément à cette "déréalisation » que nous sommes confrontés en Algérie lorsqu'on enseigne les textes de la littérature française aux débutants en classe de FLE. L'intégration des textes d'auteurs algériens en classe de FLE, selon la démarche décrite plus haut, permet de faire référence à un environnement familier de l'apprenant, à même d'optimiser ses capacités d'apprentissage.

De plus, le profil de la plupart des écrivains algériens, dont Djebar, permet de supposer que l'utilisation de leurs œuvres contribuera assurément au développement de la compétence plurilingue, mais aussi et surtout, pluriculturelle; et ce, dans les situations didactiques qui concernent tous les publics, algériens et autres. 


\section{Pour une didactisation des nouvelles de Femmes d'Alger dans leur appartement}

7 Pour illustrer les avantages du recours aux textes d'auteurs algériens, nous avons choisi le recueil de nouvelles Femmes d'Alger dans leur appartement d'Assia Djebar (publiées sous forme de recueil en 2002).

Nos propositions s'inscrivent dans le cadre de l'enseignement par genres textuels, dans le dispositif de la séquence didactique et du projet- apprenant.

L'un des théoriciens de la notion de " genre », J. M. Adam, s'explique quant au choix de cette notion par rapport à celle, antérieure, de «type » :

En dépit de ce que j'ai pu écrire encore au début des années 1980, sous l'influence des travaux anglo-saxons, pour moi, d'un point de vue épistémologique et théorique, le concept de types de textes est plus un obstacle méthodologique qu'un outil heuristique (...). Je conclurai en insistant sur le fait que les classements par les genres me paraissent plus pertinents. $(2005: 22)$

Nous userons de la notion de « genre » dans cet esprit.

Pour le «projet-apprenant ", il est à distinguer du "projet didactique » tel que défini par Huber $(2005: 17)$ : «le projet didactique est élaboré par chaque enseignant, qui détermine quels sont les savoirs à enseigner à partir des programmes (disciplinaires) et la façon de les exposer aux élèves ».

Le projet-apprenant désigne une "une tâche, définie et réalisée en groupe (...) aboutissant à un résultat concret, matérialisable et communicable présentant une utilité par rapport à l'extérieur » (Le Grain, cité dans Pierret-Hannecart \& Pierret, 2005 : 73).

Cette tâche est différente de la simple production écrite qui clôt une séquence didactique (comme dans le cas du projet didactique). Le projet apprenant débouche sur un impact concret dans le réel : une exposition, un spectacle, une semaine culturelle, la commémoration d'un événement national, une campagne d'information, une journée d'étude, un hommage à une personnalité, etc.

Il implique donc la mobilisation de diverses compétences, souvent pluridisciplinaires. Son principal avantage réside dans le fait que "l'élève se mobilise et trouve du sens à ses apprentissages dans une production à portée sociale qui le valorise » (Huber, 1999 : 18).

\subsection{Dimensions avantageuses de la nouvelle}

8 L'œuvre Femmes d'Alger dans leur appartement est composée de sept (7) nouvelles dont les dimensions sont très différentes, comme indiqué dans le tableau ci-dessous :

\begin{tabular}{|c|l|c|c|}
\hline $\mathrm{N}^{\circ}$ & \multicolumn{1}{|c|}{ Titre de la nouvelle } & Pagination & Nombre de pages \\
\hline 1 & « La nuit du récit de Fatima » & $15-57$ & 43 \\
\hline 2 & « Femmes d'Alger dans leur appartement » & $61-130$ & 70 \\
\hline 3 & « La femme qui pleure » & $131-138$ & 08 \\
\hline 4 & « Il n'y a pas d'exil » & $141-160$ & 20 \\
\hline 5 & «Les morts parlent » & $161-217$ & 57 \\
\hline
\end{tabular}




\begin{tabular}{|l|l|c|c|}
\hline 6 & «Jour de Ramadhan » & $218-223$ & 06 \\
\hline 7 & « Nostalgie de la Horde» & $224-234$ & 11 \\
\hline
\end{tabular}

Pour classer ces nouvelles selon leurs dimensions, nous nous référons aux critères proposés par Carrara (2014). Elle en distingue trois catégories: "l'histoire courte » (moins de 7500 mots), «la novelette» (entre 7500 et 17499 mots) et «la novella, presque un roman ", (entre 17500 et 40000 mots).

Ces trois catégories de nouvelles sont représentées dans l'œuvre de Djebar :

- - «l'histoire courte »: no 3, 4, 6 et 7 ;

- - «la novelette $»: \mathrm{n}^{\mathrm{o}} 1$ et 5 ;

- - « la novella » : nº 2 .

Cette variété, qui offre la possibilité d'exploiter ces nouvelles avec des apprenants d'âges et de niveaux différents, permet de tenir compte des motivations des apprenants $^{22}$.

\subsection{Diversité des thèmes}

Parmi les divers thèmes des nouvelles, dominent ceux inscrits dans la société algérienne entre tradition et modernité, comme celui de la femme, du couple, de l'amour et du désir, de la mort, de la religion, de la guerre.

Ces thèmes sociaux, familiers de l'apprenant, sont à même de favoriser à la fois le processus ternaire de transfert des connaissances, tel que décrit plus haut, et la motivation.

Par ailleurs, la nature de ces thèmes contribue à l'établissement de liens avec un projetapprenant interdisciplinaire (qui pourrait concerner autant la matière de la langue française que d'autres) ${ }^{23}$.

Cette caractéristique commune joue en faveur de la didactisation des nouvelles de Djebar dans la mesure où elle favorise les projets interdisciplinaires. Ainsi, pour la célébration de la journée mondiale de la femme, il est possible de concevoir un projet interdisciplinaire qui va porter sur la condition de la femme et sur sa place dans la société.

Des extraits de ces nouvelles étudiées en classe peuvent alors alimenter le projet et être proposés à la lecture par ceux auxquels le produit du projet (exposition, journal mural, table ronde, etc.) est destiné.

Ce type de projet repose sur la réutilisation des acquis d'une séquence didactique qui porte, ici, sur la nouvelle dans le cadre d'un projet interdisciplinaire; ce qui contribue d'une part à pratiquer les apprentissages et à leur donner sens dans la réalité, et d'autre part à tisser des liens entre les apprentissages de différentes matières scolaires, ainsi décloisonnés.

A ces possibilités s'ajoutent celles qui peuvent s'inscrire dans le cadre d'une didactique intégrée et convergente parce que, dans l'Éducation Nationale, la nouvelle est également enseignée dans les matières en langues arabe et tamazight. Ces pratiques permettraient le transfert des acquis d'une matière vers une autre et le développement de la compétence plurilingue.

Inscrire la séquence qui porte sur la nouvelle dans un projet qui s'inspire des principes de la didactique intégrée peut par ailleurs constituer l'une des meilleures manières de 
remédier au problème que posent les productions initiale et finale dans les séquences consacrées aux genres littéraires (cf. supra).

La solution pourrait consister alors à proposer par exemple, comme technique visant à contribuer à rendre la tâche réalisable, la traduction de la nouvelle dans une langue que maîtrise quelque peu l'apprenant, à savoir la langue arabe pour le cycle primaire, l'anglais ou tamazight pour les cycles supérieurs.

\subsection{Caractéristiques linguistiques et discursives des nouvelles}

Dans une séquence didactique, les modules portent sur différents aspects linguistiques et discursifs, ici du genre sur lequel elle s'articule. La sélection des aspects qui feront l'objet de l'apprentissage se fait après une évaluation diagnostic (production initiale) pour cerner les besoins des apprenants.

Une fois la liste des contenus à enseigner établie, l'étape suivante consiste à choisir les supports - du même genre textuel - dont les caractéristiques correspondent aux contenus retenus pour les « modules » ou « ateliers».

L'étude des caractéristiques linguistiques et discursives des nouvelles de Djebar permet de constater qu'elles offrent la possibilité de travailler les contenus suivants :

- l'articulation narration/discours; la transposition narration/discours et vice-versa; l'articulation narration/description;

- la présentation d'un dialogue ; l'introduction de propos (au style direct notamment);

- la description (réaliste) du physique, du comportement, de la tenue vestimentaire et du caractère des personnages ;

- l'insertion de faits d'Histoire dans un texte littéraire ;

- l'expression des sentiments (amour, colère, douleur psychique, déception, etc.);

- les noms des métiers (surtout traditionnels);

- l'expression du temps (antériorité, simultanéité, postériorité); la concordance des temps dans le système du passé (plus que parfait/imparfait/passé simple);

- la caractérisation du style (personnification, métaphore, l'ironie, etc.); l'expression de l'implicite et du sous-entendu ;

- la traduction de l'arabe vers le français; l'expression de la médiation linguistique et culturelle ;

- la présence de l'oral dans l'écrit.

On peut en déduire que les caractéristiques linguistiques et discursives des nouvelles de Djebar permettent de prendre en charge la quasi-totalité des contenus auxquels on pourrait penser en concevant une séquence sur la nouvelle littéraire.

\section{Pour le développement de la compétence plurilingue et pluriculturelle}

11 La lecture des nouvelles de Djebar nous permet de constater que l'empreinte de l'Algérie est présente par la nature des faits et des «tableaux » décrits, mais aussi par le recours très fréquent à de nombreux termes et expressions, traduits ou pas, empruntés principalement à l'arabe et à tamazight et renseignant sur des pratiques sociales et culturelles algériennes. C'est cette dimension des textes qui autorise de les proposer pour le développement de la compétence plurilingue et pluriculturelle. Son intérêt se 
justifie par le fait qu'on la considère au cœur même de la compétence à communiquer (Conseil de l'Europe, $2001: 129$ ).

Nos propositions s'appuient sur un postulat de la définition de cette compétence : Poser qu'un même individu ne dispose pas d'une collection de compétences à communiquer distinctes et séparées suivant les langues dont il a quelque maîtrise, mais bien d'une compétence plurilingue et pluriculturelle qui englobe l'ensemble du répertoire langagier à disposition. (idem.)

Assia Djebar recourt fréquemment dans ses nouvelles à un vocabulaire particulier : le vocabulaire nécessaire pour nommer les pratiques, les us, les objets, etc., qui caractérisent l'Algérie comme dans les exemples suivants :

- « les femmes et les jeunes filles allaient à la fontaine, les premières portant des outres, les jeunes filles des cruches" (Djebar, $2002: 12$ );

- «(...) au coin d'un petit feu, d'un kanoun plein de braises sur le point de s'éteindre» (p. 15);

- "elle trayait les chèvres, allait au gaulage des olives» (p. 19).

Nous pouvons constater que des termes peu courants, appartenant à la langue étrangère, comme "gaulage " par exemple, se distribue dans un environnement rendu familier soit par l'évocation de coutumes ou de mots connus de l'apprenant, comme « kanoun ». Cette concomitance du connu et l'inconnu permet à l'apprenant d'enrichir son vocabulaire en langue étrangère en appréhendant le second en s'aidant des repères du premier: le rituel féminin de la fontaine, ou celui de la récolte des olives, lui facilitera la compréhension et l'acquisition du vocabulaire correspondant : « outres » « cruches » et « traire » « gaulage».

Les textes de Djebar - ou d'autres auteurs algériens qui traitent de l'Algérie et de sa culture et/ou qui recourent à des termes empruntés à ses langues - sont un moyen d'acquérir un vocabulaire en situation.

En outre, la découverte de ce vocabulaire renforce la compétence de traduction des apprenants, composante essentielle de la compétence plurilingue et pluriculturelle. Dans les textes, certains passages affichent l'opération de traduction, comme dans « elle se nommait elle-même (...) : "Itima, je suis l'itima !" - orpheline, je suis l'orpheline !» (p.19).

Dans d'autres passages, la traduction est implicite: elle est signalée par des indices discursifs (souvent inclus dans la narration), comme dans «Ma fille, à six ans, elle ira à l'école des Français: c'est décidé! (...). Cependant, à la phrase lancée si fort en arabe, l'oncle Hassan avait sursauté» (p. 32).

Le fait que ces passages traduits ne soient pas accompagnés de leurs équivalents en langue source, comme c'est le cas dans les exemples précédents, peut permettre une activité qui consisterait à chercher l'énoncé premier à partir duquel la traduction est censée être obtenue.

Les passages que nous avons cités jusqu'à présent visaient davantage le développement de la compétence plurilingue. Nous en avons repéré d'autres qui favoriseraient la compétence pluriculturelle, même s'il est difficile pour nous d'établir une frontière entre les deux, étant donné le lien étroit qu'entretient la langue avec la culture.

$\mathrm{Si}$ ce dernier objectif concerne surtout les apprenants d'autres cultures, il peut également concerner les Algériens dans la mesure où certaines des pratiques décrites diffèrent d'une région à une autre; de ce fait, elles peuvent être inconnues de l'apprenant.

Afin de développer la compétence pluriculturelle, on peut se contenter de faire découvrir aux apprenants, grâce à ces passages textuels, les pratiques sociales ou culturelles qui caractérisent une communauté algérienne donnée. 
Il en est ainsi :

- du rituel de la circoncision : «Ce soir, veille de la circoncision de notre dernier - que Dieu nous le garde! Pour sa protection et son bonheur futur, nous lui mettrons un peu de henné sur les mains» (p. 90) ;

- de l'évocation du rituel de la prière : «C'était un vendredi, (...) d'un minaret et d'une mosquée proche, le muezzin (...) appelait les fidèles à la prière solennelle du milieu du jour» (p. 54) ;

- de la présentation de la religion chrétienne : "Le christianisme est adoration de la Vierge mère » (p.250) ;

- de la description de costumes traditionnels: «(...) habillés à l'arabe, à l'ancienne: avec un pantalon bouffant et filet de serge dorée sur les coutures, un burnous blanc de laine posé sur une épaule et un fez rouge au-dessus de leurs cheveux frisés " (p.30); etc.

Quand ces pratiques décrites sont très différentes de celles des apprenants, l'enseignant devrait alors les conduire à les comprendre en établissant des passerelles vers leur propre(s) culture(s). Cela favoriserait la compréhension, et contribuerait aussi à développer l'esprit d'ouverture vers les autres cultures.

Au terme de cette modeste étude, rappelons que penser à la didactisation des nouvelles d'Assia Djebar nous a tout d'abord confronté aux problèmes que pose l'enseignement des ou par des textes issus des littératures francophones, dont celui relatif au caractère authentique de ces productions. Nous avons tenté de montrer que ces textes étaient des documents authentiques à part entière et que leur didactisation était conforme aux exigences aussi bien de la didactique institutionnelle que de celle des chercheurs. Nous en avons déduit que le recours à ces textes d'auteurs algériens était d'autant plus nécessaire qu'ils semblent être un moyen de favoriser le développement de la compétence plurilingue et pluriculturelle qui est au cœur des approches didactiques actuelles, dont l'approche actionnelle.

\section{BIBLIOGRAPHIE}

ADAM J. M., « La notion de typologie de textes en didactique du français : une notion " dépassée" ? », in. Recherches, № 42, pp. 11-23, 2005. Disponible sur : http://www.revue-recherches.fr/wp-content/uploads/2014/06/42_011-023__adam_.pdf ADAMI H., «Les documents authentiques dans la formation des adultes migrants : pratiques pédagogiques et contraintes institutionnelles ", Mélanges CRAPEL, no 31, 2009, pp. 159- 172.

BAILLY D., Didactique de l'anglais. 1. Objectifs et contenus de l'enseignement, Paris, Nathan pédagogie, 1997.

BOUCHARD R., « Documents authentiques oraux et transposition didactique ». Consulté sur le site :

< https://hal.archives-ouvertes.fr/hal-00377026 >

BOUCHOUIKA F. et al., Français $3^{e}$ A.S., Alger, IPN, 1989.

BOUCHOUIKA F. et al., Livre de Français $3^{\text {ème }}$ AS, Alger, ONPS, 2004. 
BRAHIMI D., Langue et littératures francophones, Paris, Ellipses, 2001.

BYRAM M., GRIBKOVA B. et STARKEY H., Développer la dimension interculturelle de l'enseignement des langues une introduction pratique a l'usage des enseignants, Conseil de l'Europe, Strasbourg 2002. Disponible sur le site :

< https://www.coe.int/t/dg4/linguistic/Source/Guide_dimintercult_FR.pdf >

CAFE-PEDAGOGIQUE, Appel «Pour l'enseignement des Littératures Francophones en France », 2006. Disponible sur le site : < http://www.cafepedagogique.net/lemensuel/lenseignant/Documents/ pdf/appelf.pdf >

CARRARA M. A., « Les 8 points essentiels de la nouvelle littéraire », 2014. Disponible sur le site : < http://www.aproposdecriture.com/les-8-points-essentiels-de-la-nouvelle-litteraire >.

CHABANE F. et al., Livre de français 2 AS, MEN/ONPS, ALGER, 2004.

COÏANIZ, A., « Apprentissage des langues en maternelle : l'entrée dans le langage en français langue non maternelle », 1998. Disponible sur le site :

< http://guyane.scola.ac-paris.fr/ftp_public/Coianiz.pdf >

CONSEIL DE L'EUROPE, Un cadre européen commun de référence pour les langues : apprendre, enseigner, évaluer, Paris, Didier, 2001.

COSTE D., « Diversité des plurilinguismes et formes de l'éducation plurilingue et interculturelle », in Les Cahiers de l'Acedle, Volume 7, No 1, Notions en question en didactique des langues, 2010,

pp. 141-165. Disponible sur le site :

$<$ http://acedle.org/IMG/pdf/Coste_Cahiers-Acedle_71.pdf >

$<$ https://journals.openedition.org/rdlc/2031 >

CUQ J. P., (dir.), Dictionnaire de didactique du français langue étrangère et seconde, Paris, Clé International, 2003.

DEFAYS J. M., DELTOUR S., Le Français langue étrangère et seconde : enseignement et apprentissage, Liège, Mardaga, 2003.

DE PIETRO J. F., «Et si, à l'école, on apprenait aussi ? Considérations didactiques sur les apports et les finalités des apprentissages langagiers guidés », in AILE, $\mathrm{n}^{\circ}$ 16, 2002. Ddisponible sur le site : $<$ http://aile.revues.org/1382 >

DJEBAR A., Femmes d'Alger dans leur Appartement, Paris, Albin Michel, 2002.

DJILALI K., Français Première année secondaire lettre, Alger, MEN/ONPS, 2005.

DOLZ J. \& SCHNEUWLY B., Pour un enseignement de l'oral : Initiation aux genres formels à l'école, Paris, ESF, 1998.

DOLZ J., NOVERRAZ M. \& SCHNEUWLY B. (dirs), S'exprimer en français. Séquences didactiques pour l'oral et pour l'écrit, Volume 4, Bruxelles, De Boeck/COROME, 2002.

GALISSON R. \& COSTE D., Dictionnaire de didactique des langues, Hachette, 1976.

HUBER M., Apprendre en projets : la pédagogie du projet-élèves, Lyon, Chronique Sociale, 1999.

HUBER M., Conduire un projet-élèves, Paris, Hachette-Éducation, 2005. LAGHA A. (2005) « Le livre scolaire : une nouvelle vision ", in Toualbi- Thaâlibi, N. \& Tawil, S. (Dirs), La Refonte de la pédagogie en Algérie - Défis et enjeux d'une société en mutation, Alger, ONPS, Casbah éditions, pp. 57-66.

MAHBOUBI F., REKKAB M. \& ALLAOUI A., Français Troisième année secondaire, Alger, MEN/ONPS, 2007. 
MEN, « Document d'accompagnement du programmes, Français $2^{\mathrm{e}}$ A.S. », Alger, Ministère de l'Éducation Nationale, 2006.

MEN, « Référentiel général des programmes », Alger, Ministère de l'Éducation Nationale, 2009a.

MEN, « Guide méthodologique d'élaboration des programmes », Alger, Ministère de l'Éducation Nationale, 2009b.

MILED M., « Un cadre conceptuel pour l'élaboration d'un curriculum selon l'approche par les compétences ", dans Toualbi-Thaâlibi, N. \& Tawil, S. (Dirs), La Refonte de la pédagogie en Algérie Défis et enjeux d'une société en mutation, Alger, ONPS, Casbah éditions, pp. 125136.

PIERRET-HANNECART M. \& PIERRET P., Des pratiques pour l'école d'aujourd'hui, Bruxelles, De Boeck, 2005.

PUREN Ch., « Variations sur le thème de l'agir social en didactique des langues-cultures étrangères », 2009. Disponible sur :

< http://www.christianpuren.com/mes-travaux/2009b/ >

RENAUD P., « Absoute pour un locuteur natif », in Le français en Afrique, no 12, 1998, pp. 257272. Disponible sur le site :

$<$ http://www.unice.fr/ILF-CNRS/ofcaf/12/12.html >

REUTER Y. (éd.) et al. Dictionnaire des concepts fondamentaux des didactiques, Bruxelles, De Boeck, 2010.

ROSIER J. M., « Réflexions didactiques sur la littérature francophone de Belgique ", in Textyles, $n^{\circ}$ 15, 1999, 166 174, 2012. Disponible sur :

< http://textyles.revues.org/1132 >

ROSIER J. M., « Le surréalisme de Belgique : un problème d'histoire littéraire ? », in Pratiques, $\mathrm{N}^{\circ}$ 93, mars 1997. Consulté sur le site :

< http://www.pratiques-cresef.com/p093_ro1.pdf >

TARDIF J. \& MEIRIEU P., « Stratégie pour favoriser le transfert de connaissances », in Vie pédagogique, Revue québécoise de développement pédagogique, N 98, mars-avril 1996, pp. 4-7.

\section{NOTES}

1. Consulté sur le site : < http://www.cafepedagogique.net/lemensuel/lenseignant/Documents/ pdf/appelf.pdf >

2. Bailly distingue la «didactique institutionnelle» (recommandations institutionnelles) de la «didactique des chercheurs» ou "des universitaires» (1997: 16-46). Concilier les propositions des deux didactiques, qui divergent souvent, est pourtant indispensable.

3. «L'approche par les compétences traduit le souci de privilégier une logique d'apprentissage centrée sur l'élève, sur ses actions et réactions face à des situations- problèmes » (MEN, 2009a : 21).

4. "L'approche curriculaire a pour but de faire converger les programmes d'études vers le destinataire unique, c'est-à-dire l'élève. Cette convergence s'appuie sur les compétences transversales, mais aussi sur des thèmes transversaux pouvant être traités par plusieurs disciplines » (MEN, 2009b : 12).

5. "La seule approche fiable, universellement admise et scientifiquement reconnue en matière d'enseignement/apprentissage des langues, est une approche intégrée, réflexive et coordonnée des langues en présence » (MEN, 2009a : 18).

6. "Une démarche de projet : est une entreprise collective gérée par le groupe-classe (...); s'oriente vers une production concrète (...) ; induit un ensemble de tâches (...) ; suscite l'apprentissage de savoirs et de savoir- 
faire de gestion de projet (...); favorise en même temps des apprentissages identifiables (au moins aprèscoup) figurant au programme d'une ou plusieurs disciplines » (MEN, 2009 b : 29).

7. «Chaque séquence est organisée autour d'un savoir-faire à maîtriser (un niveau de compétence) », dans Document d'accompagnement du programmes, Français $2^{\text {ème }}$ A.S., $2006: 4$.

8. Cette compétence est ainsi définie dans le Cadre Européen Commun de Référence pour les Langues (CECRL): «On désignera par compétence plurilingue et pluriculturelle, la compétence à communiquer langagièrement et à interagir culturellement d'un acteur social qui possède, à des degrés divers, la maîtrise de plusieurs langues et l'expérience de plusieurs cultures » (Conseil de l'Europe, 2001 : 129).

9. Cf. Jean-Maurice Rosier, «Réflexions didactiques sur la littérature francophone de Belgique », Textyles [En ligne], 15 | 1999, mis en ligne le 19 juillet 2012, consulté le 04 janvier 2016. URL : < http://textyles.revues.org/1132 >

10. Nous comprenons "séquence didactique" au sens de "suite de modules d'enseignement organisés conjointement afin d'améliorer une pratique langagière déterminée » (Dolz \& Schneuwly, $1998: 57)$.

11. Jean-François De Pietro, "Et si, à l'école, on apprenait aussi ?", Acquisition et interaction en langue étrangère [En ligne], 16 | 2002, mis en ligne le 16 décembre 2005, URL: < http:// aile.revues.org/1382 >

12. On serait confronté au même obstacle si l'on optait pour le modèle classique ternaire de la séquence didactique : Compréhension - Activités de langue - Production.

13. Dans l'actuel manuel de $1^{\text {ère }}$ AS (Djilali, 2004), on ne trouve qu'un texte de Mouloud Feraoun, un de Jean Amrouche, un paragraphe d'un texte de Mohammed Dib et un extrait d'un texte de Taous Amrouche. Dans celui de la $2^{\text {ème }}$ AS (Chabane et al., 2004), il n'y a que deux paragraphes : l'un de Malek Haddad et l'autre de Mouloud Mammeri.

14. Robert Bouchard, "Documents authentiques oraux et transposition didactique ». Consulté sur le site : < https://hal.archives-ouvertes.fr/hal-00377026 >

15. La situation à laquelle renvoie ce dernier objectif concerne la société algérienne.

16. L'approche actionnelle considère que les apprenants sont "des acteurs sociaux ayant à accomplir des tâches (...) dans des circonstances et un environnement donnés, à l'intérieur d'un domaine d'action particulier » et que les activités langagières s'inscrivent "à l'intérieur d'actions en contexte social qui seules leur donnent leur pleine signification » (Conseil de l'Europe, 2001 :15).

17. "Dans son acception anglo-saxonne, le curriculum désigne la conception, l'organisation et la programmation des activités d'enseignement/apprentissage selon un parcours éducatif. Il regroupe l'énoncé des finalités, les contenus, les activités et les démarches d'apprentissage, ainsi que les modalités et moyens d'évaluation des acquis des élèves", Mohammed Miled, "Un cadre conceptuel pour l'élaboration d'un curriculum selon l'approche par les compétences ", dans La refonte de la pédagogie en Algérie - Défis et enjeux d'une société en mutation, Alger, UNESCO-ONPS, pp. 125-136. Disponible sur le site : < http://www.bief.be/docs/divers/elaboration_de_cv_070110.pdf >

18. Dans l'article de Patrick Renaud que cite D. Coste et intitulé «Absoute pour un locuteur natif", l'auteur veut "essayer de comprendre pourquoi une catégorie dont chacun s'accorde à reconnaître qu'elle ne correspond à rien "dans la réalité" continue sa carrière envers et contre tout dans les discours savants et ordinaires. Quels services irremplaçables rend-elle donc (...) ? (...) pourquoi, en matière de langage, introduire une "disposition de naissance" permettant de construire deux classes de locuteurs: les natifs et les autres? ». Il conclut son article sur la récusation du terme " natif»: «Ce qu'il faut retenir c'est que le trait de nativité est illusoire et pervers: il opacifie et réifie de part et d'autre du mur d'authenticité qu'il installe pour les séparer, activités de participation où s'investissent les répertoires verbaux et activités d'interprétation où se construisent et s'exhibent "à toutes fins pratiques" différences et convergences entre participants, avec leurs enjeux identitaires. Il faut donc repenser l'opposition entre vernaculaire et véhiculaire et en chercher éventuellement les sources, comme nous l'avons suggéré, dans les conditions socio-culturelles qui configurent en situations diverses, entre pidgin à l'état naissant et langue 
morte, l'espace social de l'activité langagière ", in Le français en Afrique, nº 12,1998, pp. 257-272. Disponible sur le site : < http://www.unice.fr/ILF-CNRS/ofcaf/12/12.html >

19. Cette théorie, traduite parfois en «Zone proximale de développement » est au cœur de la théorie socioconstructiviste de Vygotski. Elle implique qu'on propose «des activités d'apprentissage suffisamment difficiles pour qu'ils [les apprenants] apprennent quelque chose, et suffisamment faciles pour qu'ils ne se découragent pas » (Coïaniz, 1998 : 8). Consulté sur le site : < http://guyane.scola.acparis.fr/ftp_public/Coianiz.pdf >.

20. J.Tardif et P. Meirieu : "Stratégie pour favoriser le transfert de connaissances", in Vie pédagogique, Revue québécoise de développement pédagogique, $\mathrm{N}^{\circ}$ 98, mars-avril 1996, pp. 4-7. Consulté sur le site : < http://w4.uqo.ca/moreau/documents/Tardif1996.pdf >

21. Rosier J. M., «Le surréalisme de Belgique : un problème d'histoire littéraire ? ", in Pratiques, $\mathrm{N}^{\circ}$ 93, mars 1997. Consulté sur le site : < http://www.pratiques-cresef.com/p093_ro1.pdf >

22. La longueur des textes n'est que l'un des critères sur lesquels on s'appuie pour le choix des textes à utiliser en classe ; il est souvent combiné à d'autres dont ceux relatifs au degré de leur lisibilité par les apprenants.

23. Dans les programmes de l'Éducation Nationale, le thème de la guerre de libération par exemple est présent aussi bien dans les supports indiqués pour l'enseignement des langues arabe et française que dans les contenus de la matière de l'Histoire.

\section{RÉSUMÉS}

Notre réflexion porte globalement sur l'intérêt de recourir aux productions littéraires francophones d'auteurs algériens dans l'enseignement du français langue étrangère. Nous voulons, à travers l'exemple des nouvelles du recueil Femmes d'Alger dans leur appartement d'Assia Djebar, montrer que la didactisation des textes de ces auteurs peut s'avérer bénéfique pour l'enseignement / apprentissage de la langue étrangère. Nous traitons d'abord de la problématique générale de la didactisation des textes d'auteurs algériens. Ensuite, nous l'inscrivons dans le cadre de la séquence didactique et du projet- apprenant. Enfin, nous formulerons des propositions quant à l'utilisation de ces nouvelles pour le développement de la compétence plurilingue et pluriculturelle.

Our thinking relates generally to the benefit of using French literary productions of Algerian writers in teaching French as a foreign language.

We want, through the example of the short stories gathered in the book Women of Algiers in their apartment of Assia Djebar, to show that the didactization of the texts of these authors is essential and can be very beneficial. We first address the overall problem of didactization of Algerian author's texts. Then we inscribe it in the context of teaching and learning sequence and project. We conclude with proposals to use the short stories for the development of multilingual and multicultural competence. 
INDEX

Keywords : didactic text, french's literatures, authentic document, short stories, multilingual competence, pluricultural competence, Algiers, Women in their Apartment, Assia Djebar Mots-clés : didactique du texte, littératures francophones, document authentique, compétence plurilingue, compétence pluriculturelle, Femmes d'Alger dans leur appartement, Assia Djebar

\section{AUTEUR}

\section{M'HAND AMMOUDEN}

Faculté des lettres et des langues Laboratoire LAILEMM Université A. Mira - Bejaia Algérie 